\title{
COMMENT
}

\section{Use of quantitative PCR assay for amphibian chytrid detection: comment on Kriger et al. $(2006 a, b)$}

\author{
Kevin G. Smith* \\ Department of Zoology, School of Environmental Sciences and Development, North-West University, \\ Private Bag X6001, Potchefstroom 2520, South Africa
}

Present address: Department of Biology, Box 1229, Washington University in St. Louis, 1 Brookings Drive, St. Louis, Missouri 63130, USA

In the short time since the discovery and description of Batrachochytrium dendrobatidis as an agent of amphibian decline, significant research has focused on the improvement of diagnostic techniques for the detection of chytrid infection in amphibians (Berger et al. 2002, Annis et al. 2004, Olsen et al. 2004, Hyatt et al. 2007, this issue). Although histological preparation and microscopic examination is still the most widely used method of detecting cutaneous chytridiomycosis in amphibians (Kriger et al. 2006a and citations within), there is justifiable concern that the sensitivity of histopathology is unacceptably low, resulting in some proportion of false negatives in chytrid surveys (e.g. Boyle et al. 2004, Olsen et al. 2004).

The search for more sensitive assays for Batrachochytrium dendrobatidis has resulted in the development of molecular techniques capable of detecting the equivalent of 10 zoospores of $B$. dendrobatidis (Annis et al. 2004), a resolution potentially fine enough to detect a single chytrid sporangium. The sensitivity envelope has been pushed even further, however, and real-time quantitative PCR (qPCR) techniques are now capable of detecting the equivalent of a single zoospore (Boyle et al. 2004). Recent analyses suggest that $\mathrm{qPCR}$ is more sensitive, more specific, and in some ways more convenient than histopathology (Kriger et al. 2006a). This result, combined with the quantitative nature of qPCR, has led Kriger et al. $(2006 \mathrm{a}, \mathrm{b})$ to recommend that amphibian disease biologists make swab-qPCR the primary method of detection of $B$. dendrobatidis.

There is no reasonable argument to be made against using $\mathrm{qPCR}$ for chytrid detection if identifying very small numbers of zoospores is of primary concern. I agree with Kriger et al. (2006a) that qPCR outperforms all other known techniques in detecting zoospores of Batrachochytrium dendrobatidis and in not falsely reporting the presence of chytrid zoospores. That is, qPCR has high sensitivity and high specificity, a valuable combination in a single diagnostic test. However, in this comment I suggest that recommending the widespread adoption of swab-qPCR as the primary method of detecting $B$. dendrobatidis is premature and ignores potentially important differences in the goals and scope of amphibian disease monitoring programs. I also propose that additional research is needed before we can confidently conclude that qPCR quantifies the severity of chytrid infections in a meaningful way.

My primary concern with the widespread adoption of qPCR as the primary chytrid diagnostic is that it is, somewhat paradoxically, too sensitive for the reliable diagnosis of chytridiomycosis. qPCR does not actually detect chytridiomycosis sensu stricto (cf. Kriger et al. 2006a,b), but detects chytrid zoospores. This is not simply a semantic issue. There is no rigorous definition of how many zoospores are representative of chytrid infection, or how few should be considered to be artifactual and not representative of infection. In many cases, for example when large numbers of zoospores are detected by qPCR, zoospore presence and chytridiomycosis are almost certainly functionally equivalent, and it is silly to argue otherwise. However, if the high sensitivity of qPCR for detecting zoospores is combined with researchers equating the detection of a few zoospores with the presence of chytridiomycosis, then the result is effectively a latent reduction of the specificity of the diagnostic test. 
Consider, for example, a situation in which researchers are interested in determining the prevalence of chytridiomycosis within a population. Because such a study is conducted at an ecologically small scale of resolution (i.e. the unit of measure is disease status of individuals), it may seem as though the most sensitive diagnostic technique, qPCR, is called for. But this is not necessarily so, and this can be illustrated by considering the data in Table 1 of Kriger et al. (2006a). This table shows that there are several cases where the presence of a very small number of zoospores results in categorizing individuals as 'chytrid positive' (6 individuals with fewer than 10 zoospore equivalents detected). In the hypothetical case in which the researchers are asking about disease prevalence, however, they are not actually interested in the presence of a few zoospores, but in the presence of the disease caused by Batrachochytrium dendrobatidis, chytridiomycosis. Do 0.4 zoospore equivalents indicate an infected individual, or simply an individual that has come into contact with a few (perhaps viable, perhaps inviable) zoospores? Is the presence of 4 zoospore equivalents representative of an infection? How about 18 zoospores? Twenty-four zoospores? Whatever the answer may be, it is essential to recognize that qPCR does not detect the disease chytridiomycosis, but instead detects chytrid zoospores. The presence of low numbers of zoospores may result in or from chytridiomycosis, but this is not necessarily so. In other words, if qPCR is uncritically adopted as the primary diagnostic method for identifying chytridiomycosis, then the use of this technique could result in frequent commission errors, errors in which uninfected individuals are incorrectly classified as infected. This is the latent reduction in specificity to which I previously referred. When used at its highest level of sensitivity for the detection of a small number of zoospores, qPCR loses its biological relevance and in some ways becomes a molecular party trick.

Admittedly, it is a luxury to quibble that a diagnostic technique is too sensitive and I agree with Kriger et al. (2006b) that a large number of applications will benefit from the use of qPCR. For example, some researchers will, in fact, be interested in detecting the absolute presence of Batrachochytrium dendrobatidis regardless of the number of zoospores detected, making qPCR not only an acceptable, but the preferred diagnostic technique. This would be the case in amphibian breeding facilities and zoos and in the search for chytrid presence in areas of high amphibian endemism such as Madagascar. In other cases, however, researchers instead will be interested in the presence of a biologically significant amount of $B$. dendrobatidis and may correctly conclude that a few zoospores do not necessarily indicate chytridiomycosis. A problem with qPCR is that we do not know what to consider a biologically significant amount of chytrid zoospores. One solution is to define 'chytridiomycosis' based on a minimum zoospore count, but until we have empirical data that define the relationship between zoospore counts and chytrid infection, such a determination would be arbitrary.

In contrast to $\mathrm{qPCR}$, histology is a far less sensitive technique, but it has the benefit of only detecting biologically significant chytrid levels, since it is dependent on the presence of clusters of zoosporangia. Such clusters are the hallmark of a chytrid infection, so a positive histological result is a robust confirmation of chytridiomycosis. If detection of chytridiomycosis is the goal of a study, histological examination is more specific than qPCR. However, as qPCR is not always appropriate, neither is histology, so it is only individual researchers who can decide on the most appropriate chytrid detection technique based on the designs and goals of their studies. I suggest for this reason that the recommendation of widespread adoption of any single method of chytrid detection is premature.

My second concern is with Kriger et al.'s (2006a) recommended use of qPCR as a method for the quantification of chytrid infection in amphibians. One of the perceived strengths of qPCR is that it can provide a count of the amount of Batrachochytrium dendrobatidis detected in zoospore equivalents, providing researchers with a quantitative measure of chytrid presence. If reliable, quantification of chytridiomycosis represents a significant advance in chytrid research, which almost certainly has been restricted by the low resolution of the binary presence/absence data provided by histology. As Kriger et al. (2006a) point out, 2 amphibian populations with a similar prevalence of chytrid infection may have significantly different average infection intensities. Accurate quantification of chytrid load in amphibian populations may help answer one of the most vexing questions about the role of $B$. dendrobatidis in amphibian declines: Why does chytrid cause mortality, extirpations, and extinctions in some species (or populations, or localities, or months) but not others? Any advance toward the quantification of chytridiomycosis in amphibians is therefore important, and the development of qPCR for this purpose is a valuable step.

However, before we adopt qPCR counts of zoospore equivalents as a measure of severity of chytridiomycosis, a number of important questions must be answered about the biology of Batrachochytrium dendrobatidis. Most importantly, the strength of the relationship between zoospore count and infection severity is not known. Indeed, we do not yet have confirmation that such a relationship exists. We also do not know if zoospore production by $B$. dendrobatidis is constant relative to severity of infection. In other words, we do not know if a heavy chytrid load always produces a representatively large number of zoospores. 
Evidence from field and laboratory studies suggests that the relationship between zoospore count and infection severity is neither simple nor constant. It is well known that the chytrid life cycle is temperature-dependent and growth and zoospore production rates vary with environmental temperature (Piotrowski et al. 2004). It follows that zoospore production and/or infection severity may vary with season, a view that is supported by the observed seasonality of chytrid-associated mortality events (Berger et al. 1998, 2004, Bosch et al. 2001), although seasonal changes in other stressors may be responsible for these patterns, as well. Ultimately, we must identify and consider factors such as these and determine the signal-to-noise ratio of qPCR zoospore counts before we can reliably consider them to be a quantitative measure of chytrid infection levels. Quantitative PCR is ideal for such studies, and by combining qPCR with thoughtfully designed laboratory experiments and field studies it should not be difficult to validate $\mathrm{qPCR}$ as a quantitative measure of chytridiomycosis. It may be profitable to combine the use of qPCR with new microscopic quantification methods (e.g. Weldon \& du Preez 2006) to validate qPCR for quantification of chytridiomycosis. This would reverse the recent pattern of using PCR to validate histology, but such a reversal may be warranted.

In summary, I agree with Kriger at al. (2006a,b) that swab-qPCR is a highly sensitive and useful technique for the study of Batrachochytrium dendrobatidis and it has the potential to result in new discoveries about the role played by this pathogen in amphibian declines. However, as with any tool, the use of qPCR is appropriate in some situations and less so in others. Rarely is there one universally superior tool. All amphibian disease biologists should carefully consider the full range of techniques available to them for the study of amphibian chytrid before adopting one for use. It is critical that researchers make these decisions in an informed way and not simply because of superlatives of performance that are not always biologically relevant.

\section{Editorial responsibility: Managing Editor}

Acknowledgements: L. H. du Preez and C. Weldon provided thoughtful comments on an earlier version of this manuscript.

\section{LITERATURE CITED}

Annis SL, Dastoor FP, Ziel H, Daszak P, Longcore JE (2004) A DNA-based assay identifies Batrachochytrium dendrobatidis in amphibians. J Wildl Dis 40:420-428

Berger L, Speare R, Daszak P, Green DE and 10 others (1998) Chytridiomycosis causes amphibian mortality associated with population declines in the rain forests of Australia and Central America. Proc Natl Acad Sci USA 95: 9031-9036

Berger L, Hyatt AD, Olsen V, Hengstberger SG, Boyle D, Marantelli G, Humphreys K, Longcore JE (2002) Production of polyclonal antibodies to Batrachochytrium dendrobatidis and their use in an immunoperoxidase test for chytrdidiomycosis in amphibians. Dis Aquat Org 48:213-220

Berger L, Speare R, Hines HB, Marantelli G and 10 others (2004) Effect of season and temperature on mortality in amphibians due to chytridiomycosis. Aust Vet J 82:31-36

Bosch J, Martinez-Solano I, Garcia-Paris M (2001) Evidence of a chytrid fungus infection involved in the decline of the common midwife toad (Alytes obstetricans) in protected areas of central Spain. Biol Conserv 97:331-337

Boyle DG, Boyle DB, Olsen V, Morgan JAT, Hyatt AD (2004) Rapid quantitative detection of chytridiomycosis (Batrachochytrium dendrobatidis) in amphibian samples using real-time Taqman PCR assay. Dis Aquat Org 60:141-148

Hyatt AD, Boyle DG, Olsen V, Boyle DB and 11 others (2007) Diagnostic assays and sampling protocols for the detection of Batrachochytrium dendrobatidis. Dis Aquat Org 73: 175-192

Kriger KM, Hines HB, Hyatt AD, Boyle DG, Hero JM (2006a) Techniques for detecting chytridiomycosis in wild frogs: comparing histology with real-time Taqman PCR. Dis Aquat Org 71:141-148

Kriger KM, Hero JM, Ashton KJ (2006b) Cost efficiency in the detection of chytridiomycosis using PCR assay. Dis Aquat Org 71:149-154

Olsen V, Hyatt AD, Boyle DG, Mendez D (2004) Co-localisation of Batrachochytrium dendrobatidis and keratin for enhanced diagnosis of chytridiomycosis in frogs. Dis Aquat Org 61:85-88

Piotrowski JS, Annis SL, Longcore JE (2004) Physiology of Batrachochytrium dendrobatidis, a chytrid pathogen of amphibians. Mycologia 96:9-15

Weldon C, du Preez LH (2006) Quantitative measure of Batrachochytrium dendrobatidis in amphibian skin. Dis Aquat Org 72:153-161

Submitted: September 30, 2006; Accepted: November 20, 2006 Proofs received from author(s): January 8, 2007 